\title{
Analytical Solution for 2D and 3D Lamb Problem in Saturated Soil Incorporating Effects of Compressibility of Solid and Pore Fluid
}

\author{
Hao XIONG*, Qingsheng CHEN, Xiaogang WANG
}

\begin{abstract}
In this paper, to avoid the complexity, a simple and efficient analytical solution is derived for both 2D and 3D Lamb problems, respectively, in saturated soil under harmonic excitations. Unlike the existing solutions, the proposed solutions for both 2D and 3D Lamb problems in saturated soil under harmonic excitation are capable of well revealing the effect of compressibility of both liquid phase and solid phase on the ground displacements. By applying Fourier transforms and Hankel transforms on the governing equations of wave propagation in saturated soil, wave equations are transformed to ordinary differential equations. Combining the boundary conditions and draining conditions on the ground surface, the displacement solutions on the surface of saturated porous soil due to line and point harmonic excitations are derived, respectively. Then, the solutions in frequency domain are obtained by inverse integral transforms. In the meanwhile, for the sake of discussion without losing its generality, the nondimensional solutions for three-dimensional Lamb problem are derived. The effectiveness and accuracy of the proposed solutions are demonstrated by employing three different approaches. Finally, parametric studies are conducted to investigate the effects of the governing parameters (i.e., exciting frequency, bulk modulus of soil matrix, and bulk modulus of pore fluid) on variation of non-dimensional displacement with the increasing distance away from the excitation source. The results indicate that, in contrast to the effect of the compressibility of soil matrix, the exciting frequency as well as the compressibility of the pore fluid play significant role in affecting the variation of displacement on ground surface subjected to excitations, which particularly highlights that the compressibility of the pore fluid should be carefully considered for evaluating the ground movements.
\end{abstract}

Keywords: compressible model; Fourier transform; Hankel transform; Lamb problem; saturated soil

\section{INTRODUCTION}

The classical problem of solutions for elastic waves generated by external forces in isotropic elastic half-space with plane surfaces was presented, (Lamb, 1904) [1], which has been called Lamb's problem. Since then, this problem has been widely used in various fields, such as foundation engineering (Liu, G. Y. et al. 2007) [2] earthquake engineering, layered elastic half-space, general anisotropy. Also, the Lamb's problem has been effectively extended to different areas, including contact mechanics and ultrasonic nondestructive evaluation (Han, J. H. et al. 2015) [3].

By means of Fourier transforms with respect to space and time variables, integral form expressions were obtained by Lamb. For the integral expressions, Cagniard (1938) [4] stated that a suitable distortion of the integration contour can lead to an exact closed expression. The Cagniard's method was then simplified (de Hoop, 1960) [5]. The foregoing technique for integration has become known as the Cagniard-de Hoop method. By applying the integral transform method and the inverse transformation technique, exact solution for a two-dimensional Lamb's problem due to a strip impulse loading in elastic media was presented (Liu, 2007) [6]. The details for solving Lamb's problem using finite element method were explored (Kravtsov, 2011) [7]. The efficiency and accuracy of integration operation were studied (Guenfoud, 2009; Arcos, 2013) [8, 9].

For anisotropic elastic solids, Kraut (1960) [10] solved the Lamb problem in a transversely isotropic solid when the surface of the half-space is parallel or perpendicular to the symmetry axis of material using Cagniard-de Hoop method (Kraut, E. A. et al. 1963) [10]. Burridge (1970) [11] applied Cagniard-de Hoop method to an anisotropic solid in the most general class. Hereafter, the Lamb problem was studied by means of different methods, such as self-similar boundary-initial value problem method (e.g. Willis (1973), Willis (1975), Wang (1996)) [12-14].
For saturated porous media, Lamb problem due to line source and point source was solved (Paul, 1976) $[15,16]$ using potential decomposition and Laplace-Fourier transforms. The complicated displacement solution was presented by means of Cagniard-de Hoop method. Since the item denoting couple effects between soil phase and fluid phase in the governing equations cannot be measured easily and the fluid viscosity is neglected with a result that the solution could not be used widely. Using Helmholtz decomposition method, Lamb problem in half-space due to harmonic loading was studied and the solution with integral form was obtained (Philippacopoulos, 1988) [17]. But Sharma (1992) [18] pointed out that the boundary conditions used by Philippacopoulos were not correct. Again the problem was solved using the foregoing method by Sharma. Lamb problem in three-dimensional nonaxisymmetrical space in transversely isotropic saturated porous media was studied by Huang et al. (2004) [19] using Helmholtz decomposition and integral transforms method. Transient Lamb problem in cylindrical coordinates in transversely isotropic saturated porous media was calculated using Laplace-Fourier-Hankel transforms (Wang, 2011) [20]. However, the compressibility of fluid phase and solid phase is not taken into account in the above existing studies. In particular, expressions of the solutions to the Lamb problem in saturated soil, such as transversely isotropic saturated porous soil, are often significantly complicated. It is well known that the compressibility of materials is a basic property. The compressibility of fluid and (or) solid is considered in some engineering problems, such as analytical solutions for damage initiation induced by pore pressure variation (Mattos, H. S. D. C. et al. 2018) [21], analysis of beams on layered poroelastic soils (Ai, Z. Y. et al. 2016) [22], and optimal control problem for flows (Tamellini, M, et al. 2018) [23]. Likewise, it is of great importance for considering the compressibility of fluid phase and solid phase in the Lamb problem.

To address the above issues, in this paper, a simple and efficient analytical solution is derived for the Lamb 
problems in saturated soil under harmonic excitation, where the compressibility of liquid phase and solid phase is considered. Considering a large number of plane strain problems in engineering, not only the solutions of 3D case but also those of $2 \mathrm{D}$ case are presented, in order to give all aspects of the problem. By applying Fourier transforms and Hankel transforms on the governing equations of wave propagation in saturated soil, the displacement solutions on the surface of saturated porous soil due to line and point harmonic excitations are derived, respectively. Furthermore, the solutions in frequency domain are obtained by inverse integral transforms. The effectiveness and accuracy of the proposed solutions is demonstrated by employing three different approaches. In the meanwhile, for the sake of discussion without losing its generality, the non-dimensional solutions for three-dimensional Lamb problem are derived. Finally, parametric studies are conducted to investigate the effects of the governing parameters (i.e., exciting frequency, bulk modulus of soil matrix, and bulk modulus of pore fluid) on variation of non-dimensional displacement with the increasing distance away from the excitation source. The results and discussion are presented in details.

\section{TWO-DIMENSIONAL LAMB PROBLEM \\ 2.1 Governing Equations}

The governing equations describing wave propagation in the porous saturated media with slightly compressible fluid, neglecting body force, can be expressed as (Biot, 1956) [24].

$$
\begin{aligned}
& G \boldsymbol{u}_{i, j j}+\left(\lambda_{c}+G\right) \boldsymbol{u}_{j, j i}+\alpha M \boldsymbol{w}_{j, j i}=\rho \ddot{\boldsymbol{u}}_{i}+\rho_{f} \ddot{\boldsymbol{w}}_{i} \\
& \alpha M \boldsymbol{u}_{j, j i}+M \boldsymbol{w}_{j, j i}=\rho \ddot{\boldsymbol{u}}_{i}+\frac{\rho_{f}}{n} \ddot{\boldsymbol{w}}_{i}+\frac{1}{\kappa} \dot{\boldsymbol{w}}_{i}
\end{aligned}
$$

where $\boldsymbol{u}$ denotes the displacement vector of solid skeleton; $\boldsymbol{w}$ denotes the relative displacement vector of the fluid with respect to solid; $\rho=\left((1-n) \rho_{\mathrm{s}}+n \rho_{f}\right)$ is the bulk density of solid-fluid mixture, $\rho_{\mathrm{s}}$ and $\rho_{f}$ are the densities of solid and pore fluid, respectively; $n$ is the porosity; $\kappa=k_{d} / \gamma_{f}, k_{d}$ is the permeability; $\gamma_{f}=\rho_{f} g, g$ is the gravitational acceleration; $\lambda_{c}=\lambda+\alpha^{2} M$, where $\lambda$ and $G$ are the Lamè constants of soil skeleton; $\alpha$ is defined as $\alpha=1-\frac{K}{K_{s}}=1-\frac{\lambda+2 G / 3}{K_{s}}$ , where $K_{\mathrm{s}}$ represents bulk modulus for the solid matrix; $M$ is defined as $M=\left(\frac{n}{K_{f}}+\frac{\alpha-n}{K_{s}}\right)^{-1}$, where $K_{f}$ represents bulk modulus of the pore fluid; For two-dimensional space, the subscript $i, j$ are equal to 1 or 2 and represent $x, y$ coordinates respectively; for three-dimensional space, the subscript $i, j$ are equal to 1,2 , or 3 and represent $x, y, z$ respectively; The single point and double point above the letter denote the first-order and second-order derivation with regard to time, respectively; summation convention is assumed over repeated indices.

The constitutive equations are expressed as follows:

$$
\begin{aligned}
& \sigma_{i j}=2 G \varepsilon_{i j}+\delta_{i j}\left(\lambda_{c} e+\alpha M \xi\right) \\
& -p_{f}=\alpha M e+M \xi
\end{aligned}
$$

where $\boldsymbol{\sigma}_{i j}$ is total stress tensor; $p_{f}$ is the pore fluid pressure; $\varepsilon_{i j}=\left(u_{i, j}+u_{j, i}\right) / 2$ is the solid matrix strain tensor; $e=u_{i, i}$ is the solid matrix volumetric strain; $\xi=w_{i, i}$ is the fluid volumetric strain with regard to relative displacement; $\delta_{i j}$ is Kronecher's delta.

By applying divergence operation to Eq. (1), the following expressions are obtained:

$$
\begin{gathered}
\left(\lambda_{c}+2 G\right) e_{j j}+\alpha M \xi_{j j}=\rho \ddot{e}+\rho_{f} \ddot{\xi} \\
\alpha M e_{j j}+M \xi_{j j}=\rho_{f} \ddot{e}+\frac{\rho_{f}}{n} \ddot{\xi}+\frac{1}{\kappa} \dot{\xi}
\end{gathered}
$$

In order to convert the time domain to frequency domain, the displacement vector $e$, and $\xi$ are written as $\bar{e} \cdot e^{i \omega t}$ , $\bar{\xi} \cdot e^{i \omega t}$ respectively, where $\bar{e}, \bar{\xi}$ denote amplitude functions of displacement in frequency domain, $i$ is imaginary unit, $t$ is time, $\omega$ means excitation frequency. Thus the Eq. (3) becomes:

$$
\begin{gathered}
\left(\lambda_{c}+2 G\right) \bar{e}_{j j}+\alpha M \bar{\xi}_{j j}=-\rho \omega^{2} \bar{e}-\rho_{f} \omega^{2} \bar{\xi} \\
\alpha M \bar{e}_{j j}+M \bar{\xi}_{j j}=-\rho_{f} \omega^{2} \bar{e}-\frac{\rho_{f}}{n} \omega^{2} \bar{\xi}+\frac{1}{\kappa} \mathrm{i} \omega \bar{\xi}
\end{gathered}
$$

These equations are the governing equations for steady state problems in compressible saturated porous media.

\subsection{General Solutions for Displacement}

To solve the Lamb problem, the general solutions for the governing equations will be derived by means of Fourier transforms. For two-dimensional problems, assuming that the functions in Eq. (4) are independent of coordinate $y$, Fourier transforms of the function $\bar{f}(x, z)$ with respect to coordinate $x$ can be defined as:

$$
\tilde{f}(p, z)=\int_{-\infty}^{+\infty} f(x, z) e^{i p x} \mathrm{~d} x
$$

Applying Fourier transforms defined above to Eq. (4) leads to:

$$
\begin{aligned}
& \left(\lambda_{c}+2 G\right)\left(-p^{2} \tilde{e}+\frac{\partial^{2} \tilde{e}}{\partial z^{2}}\right)+\alpha M\left(-p^{2} \tilde{\xi}+\frac{\partial^{2} \tilde{\xi}}{\partial z^{2}}\right)= \\
& =-\rho \omega^{2} \tilde{e}-\rho_{f} \omega^{2} \tilde{\xi} t \\
& \alpha M\left(-p^{2} \tilde{e}+\frac{\partial^{2} \tilde{e}}{\partial z^{2}}\right)+M\left(-p^{2} \tilde{\xi}+\frac{\partial^{2} \tilde{\xi}}{\partial z^{2}}\right)= \\
& =-\rho_{f} \omega^{2} \tilde{e}-\frac{\rho_{f}}{n} \omega^{2} \tilde{\xi}+\frac{\gamma_{f}}{k_{d}} i \omega \tilde{\xi}
\end{aligned}
$$


Subtracting $\alpha$ Eq. (6b) from Eq. (6a), the expression for $\bar{\xi}$ can be written as:

$\tilde{\xi}=\frac{1}{b_{0}}\left(\rho_{0} \omega^{2}-M_{0} p^{2}\right) \tilde{e}+\frac{M_{0}}{b_{0}} \frac{\partial^{2} \tilde{e}}{\partial z^{2}}$

where $M_{0}=\lambda+2 G, b_{0}=\alpha a_{0}-\rho_{f} \omega^{2}, \rho_{0}=\rho-\alpha \rho_{f}$, $a_{0}=\left(\rho_{f} / n\right) \omega^{2}-i\left(\gamma_{f} / k_{d}\right) \omega$.

Substituting Eq. (7) into Eq. (6b) gives an ordinary differential equation as follows:

$\frac{\mathrm{d}^{4} \tilde{e}}{\mathrm{~d} z^{4}}+\beta_{1} \frac{\mathrm{d}^{2} \tilde{e}}{\mathrm{~d} z^{2}}+\beta_{2} \tilde{e}=0$

where $\beta_{1}=-2 p^{2}+\alpha_{1}, \beta_{2}=-p^{4}+\alpha_{1} p^{2}+\alpha_{3}$,

$\alpha_{1}=\frac{\alpha b_{0}+\omega^{2} \rho_{0}}{M_{0}}+\frac{a_{0}}{M}, \alpha_{3}=\frac{\left(\rho_{f} b_{0}+a_{0} \rho_{0}\right) \omega^{2}}{M M_{0}}$.

Considering the radiation conditions of waves, the solution of Eq. (8) can be obtained:

$\tilde{e}=A_{1} \mathrm{e}^{-c z}+A_{2} \mathrm{e}^{-d z}$

where $A_{1}$ and $A_{2}$ are arbitrary functions with respect to $p, c^{2}$ and $d^{2}$ are satisfied with equation $x^{2}+\beta_{1} x+\beta_{2}=0$, and $\operatorname{Re}(c)>0, \operatorname{Re}(d)>0$. Hence, $c^{2}$ and $d^{2}$ can be expressed as:

$c^{2}=p^{2}-p_{1}^{2}$

$d^{2}=p^{2}-p_{2}^{2}$

where $p_{1}^{2}=\left(\alpha_{1}+\alpha_{2}\right) / 2, p_{2}^{2}=\left(\alpha_{1}-\alpha_{2}\right) / 2$,

$\alpha_{2}=\sqrt{\alpha_{1}^{2}-4 \alpha_{3}}$.

Substituting Eq. (9) into Eq. (7), the following equation can be obtained:

$$
\begin{aligned}
& \tilde{\xi}=\frac{1}{b_{0}}\left[A_{1}\left(\rho_{0} \omega^{2}-M_{0} p_{1}^{2}\right) \mathrm{e}^{-c z}+\right. \\
& \left.+A_{2}\left(\rho_{0} \omega^{2}-M_{0} p_{2}^{2}\right) \mathrm{e}^{-d z}\right]
\end{aligned}
$$

From Eq. (1), equations can be given as:

$$
\begin{aligned}
& G\left(\frac{\partial^{2} \bar{u}_{z}}{\partial x^{2}}+\frac{\partial^{2} \bar{u}_{z}}{\partial z^{2}}\right)+\left(\lambda_{c}+G\right) \frac{\partial \bar{e}}{\partial z}+\alpha M \frac{\partial \bar{\xi}}{\partial z}= \\
& =-\rho \omega^{2} \bar{u}_{z}-\rho_{f} \omega^{2} \bar{w}_{z} \\
& \alpha M \frac{\partial \bar{e}}{\partial z}+M \frac{\partial \bar{\xi}}{\partial z}= \\
& =-\rho_{f} \omega^{2} \bar{u}_{z}-\frac{\rho_{f}}{n} \omega^{2} \bar{w}_{z}+i \omega \frac{\gamma_{f}}{k_{d}} \bar{w}_{z}
\end{aligned}
$$

Again, applying Fourier transforms defined in Eq. (4) to Eq. (12) gives:

$$
\begin{aligned}
& G\left(-p^{2} \tilde{u}_{z}+\frac{\partial^{2} \tilde{u}_{z}}{\partial z^{2}}\right)+\left(\lambda_{c}+G\right) \frac{\partial \tilde{e}}{\partial z}+\alpha M \frac{\mathrm{d} \tilde{\xi}}{\mathrm{d} z}= \\
& =-\rho \omega^{2} \tilde{u}_{z}-\rho_{f} \omega^{2} \tilde{w}_{z} \\
& \alpha M \frac{\partial \tilde{e}}{\partial z}+M \frac{\partial \tilde{\xi}}{\partial z}=-\rho_{f} \omega^{2} \tilde{u}_{z}-\frac{\rho_{f}}{n} \omega^{2} \tilde{w}_{z}+i \omega \frac{\gamma_{f}}{k_{d}} \tilde{w}_{z}
\end{aligned}
$$

Subtracting $\alpha \cdot E q$. (13b) from Eq. (13a) leads to:

$$
\tilde{w}_{z}=\frac{1}{b_{0}}\left[\left(\rho_{0} \omega^{2}-G p^{2}\right) \tilde{u}_{z}+\left(M_{0}-G\right) \frac{\partial \tilde{e}}{\partial z}+G \frac{\partial^{2} \tilde{u}_{z}}{\partial z^{2}}\right]
$$

Then substituting Eq. (14) into Eq. (13a) gives another ordinary differential equation:

$$
\frac{\mathrm{d}^{2} \tilde{u}_{z}}{\mathrm{~d} z^{2}}-j^{2} \tilde{u}_{z}=A_{1} c \mathrm{e}^{-c z} g_{1}+A_{2} \mathrm{de}^{-d z} g_{2}
$$

where $j^{2}=p^{2}-s^{2}, s^{2}=\left(\rho \omega^{2}+\frac{\rho_{f} \rho_{0}}{b_{0}} \omega^{4}\right) / H$,

$$
\begin{aligned}
& g_{i}=\left[\left(\lambda_{c}+G\right)+\frac{\rho_{f} \omega^{2}}{b_{0}}(\lambda+G)+\frac{\alpha M}{b_{0}}\left(\rho_{0} \omega^{2}-M_{0} p_{i}^{2}\right)\right] / H, \\
& H=G\left(1+\frac{\rho_{f} \omega^{2}}{b_{0}}\right),(i=1,2) .
\end{aligned}
$$

According to Eq. (15), $\tilde{u}_{z}$ can be solved as follows:

$$
\tilde{u}_{z}=\frac{c g_{1}}{c^{2}-j^{2}} A_{1} \mathrm{e}^{-c z}+\frac{\mathrm{d} g_{2}}{d^{2}-j^{2}} A_{2} \mathrm{e}^{-d z}+A_{3} \mathrm{e}^{-j z}
$$

where $A_{3}$ is the arbitrary function with respect to $p$.

Substituting Eq. (9) and Eq. (16) into Eq. (14) yields

$$
\begin{aligned}
& \tilde{w}_{z}=\frac{1}{b_{0}}\left[A_{1} c e^{-c z}\left(\frac{g_{1}}{c^{2}-j^{2}}\left(\rho_{0} \omega^{2}-G p^{2}+G c^{2}\right)-(\lambda+G)\right)+\right. \\
& +A_{2} d \mathrm{e}^{-d z}\left(\frac{g_{2}}{d^{2}-j^{2}}\left(\rho_{0} \omega^{2}-G p^{2}+G d^{2}\right)-(\lambda+G)\right)+ \\
& \left.+A_{3} \mathrm{e}^{-j z}\left(\rho_{0} \omega^{2}-G p^{2}+G j^{2}\right)\right]
\end{aligned}
$$

Considering $\mathrm{e}=\frac{\partial u_{x}}{\partial x}+\frac{\partial u_{z}}{\partial z}, \xi=\frac{\partial w_{x}}{\partial x}+\frac{\partial w_{z}}{\partial z}$, then applying the Fourier transforms to both sides of these equations, the following equations can be obtained:

$\tilde{u}_{x}=\frac{i}{p}\left(\tilde{e}-\frac{\mathrm{d} \tilde{u}_{z}}{\mathrm{~d} z}\right)$ 
$\tilde{w}_{x}=\frac{\mathrm{i}}{p}\left(\tilde{\xi}-\frac{\mathrm{d} \tilde{w}_{z}}{\mathrm{~d} z}\right)$

Then substituting Eq. (7), Eq. (9), Eq. (16) and Eq. (17) into Eq. (18) gives:

$$
\begin{aligned}
\tilde{u}_{x}= & \frac{\mathrm{i}}{p}\left(A_{1} \mathrm{e}^{-c z}\left(1+\frac{c^{2} g_{1}}{c^{2}-j^{2}}\right)+A_{2} \mathrm{e}^{-c t}\left(1+\frac{d^{2} g_{1}}{d^{2}-j^{2}}\right)+A_{3} j \mathrm{e}^{-j z}\right) \\
\tilde{w}_{x}= & \frac{\mathrm{i}}{p b_{0}}\left[A _ { 1 } \mathrm { e } ^ { - c z } \left(\frac{c^{2} g_{1}}{c^{2}-j^{2}}\left(\rho_{0} \omega^{2}-G p^{2}+G c^{2}\right)\right.\right. \\
& \left.+\left(\rho_{0} \omega^{2}-M_{0} p^{2}+G c^{2}\right)\right)+A_{2} \mathrm{e}^{-d z}\left(\frac{d^{2} g_{1}}{d^{2}-j^{2}}\right. \\
& \left.\left(\rho_{0} \omega^{2}-G p^{2}+G d^{2}\right)+\left(\rho_{0} \omega^{2}-M_{0} p^{2}+G d^{2}\right)\right) \\
& \left.+A_{3} j \mathrm{e}^{-j z}\left(\rho_{0} \omega^{2}-G p^{2}+G j^{2}\right)\right]
\end{aligned}
$$
cation.

The following parameters are introduced for simplifi-

$$
\begin{aligned}
& \lambda_{1}=\left(c^{2}-j^{2}\right) / g_{1}, \lambda_{2}=\left(d^{2}-j^{2}\right) / g_{2}, \\
& \psi_{1}=\rho_{0} \omega^{2}-G p^{2}+G c^{2}, \psi_{2}=\rho_{0} \omega^{2}-G p^{2}+G d^{2}, \\
& \psi_{3}=\rho_{0} \omega^{2}-G p^{2}+G j^{2}, \quad \phi_{1}=\rho_{0} \omega^{2}-M_{0} p^{2}+G c^{2}, \\
& \phi_{2}=\rho_{0} \omega^{2}-M_{0} p^{2}+G d^{2}, \delta_{1}=\left(\psi_{1}-\lambda_{1}((\lambda+G)) / b_{0},\right. \\
& \delta_{2}=\left(\psi_{2}-\lambda_{2}((\lambda+G)) / b_{0}, \delta_{3}=\psi_{3} / b_{0},\right. \\
& \gamma_{1}=\left(\lambda_{1}+c^{2}\right) / p, \gamma_{2}=\left(\lambda_{2}+d^{2}\right) / p, \\
& \eta_{1}=\left(c^{2} \psi_{1}+\lambda_{1} \phi_{1}\right) /\left(p b_{0}\right), \eta_{2}=\left(d^{2} \psi_{2}+\lambda_{2} \phi_{2}\right) /\left(p b_{0}\right), \\
& A_{1}^{*}=A_{1} g_{1} /\left(c^{2}-j^{2}\right), A_{2}^{*}=A_{2} g_{2} /\left(d^{2}-j^{2}\right), A_{3}^{*}=A_{3} / p
\end{aligned}
$$

Therefore Eq. (16), Eq. (17), Eq. (19) and Eq. (20) can be written as:

$\tilde{w}_{z}=A_{1}^{*} c \delta_{1} \mathrm{e}^{-c z}+A_{2}^{*} d \delta_{2} \mathrm{e}^{-d z}+A_{3}^{*} p \delta_{3} \mathrm{e}^{-j z}$

$\tilde{u}_{z}=A_{1}^{*} c \mathrm{e}^{-c z}+A_{2}^{*} d \mathrm{e}^{-d z}+A_{3}^{*} p \mathrm{e}^{-j z}$

$$
\tilde{u}_{x}=\mathrm{i}\left[A_{1}^{*} \gamma_{1} \mathrm{e}^{-c z}+A_{2}^{*} \gamma_{2} \mathrm{e}^{-d z}+A_{3}^{*} j \mathrm{e}^{-j z}\right]
$$

$$
\tilde{u}_{x}=\mathrm{i}\left[A_{1}^{*} \eta_{1} \mathrm{e}^{-c z}+A_{2}^{*} \eta_{2} \mathrm{e}^{-d z}+A_{3}^{*} \delta_{3} j \mathrm{e}^{-j z}\right]
$$

Thus, the above equations are the general solutions for governing equations for wave propagation in the porous saturated media in two-dimensional space.

\subsection{Boundary Conditions and Special Solutions}

The arbitrary functions, such as $A_{1}^{*}, A_{2}^{*}$ and $A_{3}^{*}$, in the Eq. (21) should be determined by boundary conditions in
Lamb problem. Then special solutions, namely Lamb problem's solutions, will be presented. Assuming the ground surface is permeable, Fig. 1 shows a schematic diagram for two-dimensional Lamb problem. The vertical concentrated harmonic force is acting on the surface at origin point. The boundary condition can be given as follows:

$$
\begin{aligned}
& \tilde{\sigma}_{z}(p, 0)=-P_{0} \\
& \tilde{\tau}_{z x}(p, 0)=0 \\
& \tilde{p}_{f}(p, 0)=0
\end{aligned}
$$

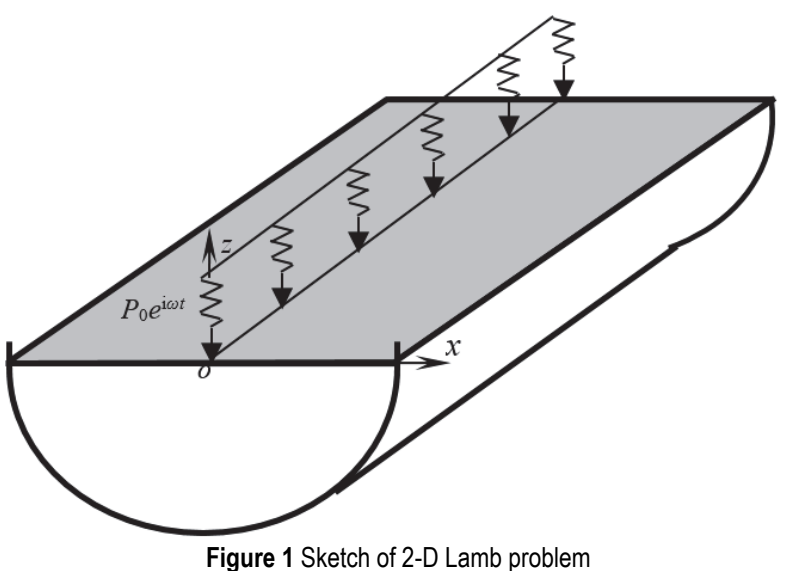

Applying Fourier transforms defined in Eq. (4) to constitutive equation Eq. (2) gives:

$\tilde{\sigma}_{z}=(\lambda+2 G) \frac{\mathrm{d} \tilde{u}_{z}}{\mathrm{~d} z}-\mathrm{i} p \lambda \tilde{u}_{x}$

$\tilde{\tau}_{z x}=G \frac{\mathrm{d} \tilde{u}_{x}}{\mathrm{~d} z}-\mathrm{i} p G \tilde{u}_{z}$

$\tilde{p}_{f}=-M\left(\frac{\mathrm{d} \tilde{w}_{z}}{\mathrm{~d} z}-\mathrm{i} p \tilde{w}_{x}\right)-\alpha M\left(\frac{\mathrm{d} \tilde{u}_{z}}{\mathrm{~d} z}-\mathrm{i} p \tilde{u}_{x}\right)$

Substituting Eq. (21) into Eq. (23) and in turn into Eq. (22) yields:

$A_{1}^{*}\left(\lambda \lambda_{1}-2 c^{2} G\right)+A_{2}^{*}\left(\lambda \lambda_{2}-2 d^{2} G\right)-A_{3}^{*}(2 G p j)=-P_{0}$

$A_{1}^{*} c\left(p+\gamma_{1}\right)+A_{2}^{*} d\left(p+\gamma_{2}\right)+A_{3}^{*}\left(j^{2}+p^{2}\right)=0$

(24b)

$$
\begin{aligned}
& A_{1}^{*}\left(c^{2}\left(\alpha+\delta_{1}\right)-p\left(\alpha \gamma_{1}+\eta_{1}\right)\right)+ \\
& +A_{2}^{*}\left(d^{2}\left(\alpha+\delta_{2}\right)-p\left(\alpha \gamma_{2}+\eta_{2}\right)\right)=0
\end{aligned}
$$

Solution of Eq. (24) gives the following expressions:

$$
A_{1}^{*}=\frac{-P_{0}}{D} \cdot\left(j^{2}+p^{2}\right) a_{2}
$$


$A_{2}^{*}=\frac{P_{0}}{D} \cdot\left(j^{2}+p^{2}\right) a_{1}$

$A_{3}^{*}=\frac{P_{0}}{D} \cdot\left(c a_{2}\left(p+\gamma_{1}\right)-d a_{1}\left(p+\gamma_{2}\right)\right)$

(25c)

where $a_{1}=c^{2}\left(\alpha+\delta_{1}\right)-p\left(\alpha \gamma_{1}+\eta_{1}\right), a_{2}=d^{2}\left(\alpha+\delta_{2}\right)-p\left(\alpha \gamma_{2}+\eta_{2}\right)$ $D=a_{1}\left(\left(j^{2}+p^{2}\right)\left(2 d^{2} G-\lambda \lambda_{2}\right)-2 d G j p\left(p+\gamma_{2}\right)-\right.$ $-a_{2}\left(\left(j^{2}+p^{2}\right)\left(2 c^{2} G-\lambda \lambda_{1}\right)-2 c G j p\left(p+\gamma_{1}\right)\right)$

Substituting Eq. (25) into Eq. (21), the displacement solutions in transformed domain for two-dimensional Lamb problem can be given by

$\tilde{u}_{z}=\frac{P_{0}}{D}\left(c a_{2}\left(p \gamma_{1}-j^{2}\right)+d a_{1}\left(j^{2}-p \gamma_{2}\right)\right)$

$\tilde{u}_{x}=\frac{\mathrm{i} P_{0}}{D}\left(a_{1}\left(j^{2}+p^{2}\right) \gamma_{2}-a_{2}\left(j^{2}+p^{2}\right) \gamma_{1}+\right.$

$+j\left(a_{2} c\left(p+\gamma_{1}\right)-a_{1} d\left(p+\gamma_{2}\right)\right)$

(26b)

Applying inverse Fourier transforms to Eq.(26), the displacement solutions in frequency domain can be expressed as:

$\bar{u}_{z}(x, 0)=\frac{1}{2 \pi} \int_{-\infty}^{+\infty} \tilde{u}_{z} \mathrm{e}^{-\mathrm{i} p x} \mathrm{~d} p$

$\bar{u}_{x}(x, 0)=\frac{1}{2 \pi} \int_{-\infty}^{+\infty} \tilde{u}_{x} \mathrm{e}^{-\mathrm{i} p x} \mathrm{~d} p$

\section{THREE-DIMENSIONAL LAMB PROBLEM \\ 3.1 General Solutions for Displacement}

The $n$-th order Hankel transforms of $F(r, z)$ with respect to $r$ is defined as:

$\hat{F}_{n}(p, z)=\int_{0}^{+\infty} r F(r, z) J_{n}(p r) \mathrm{d} r$

where $J_{n}(p r)$ is the Bessel function of the first kind of order $n$.

Utilizing symmetrical cylindrical coordinate in threedimensional space, and applying Hankel transforms instead of Fourier transforms to Eq. (4), the displacement solutions can be obtained as follows:

$$
\begin{aligned}
& \hat{w}_{z 0}=A_{1}^{*} c \delta_{1} \mathrm{e}^{-c z}+A_{2}^{*} d \delta_{2} \mathrm{e}^{-d z}+A_{3}^{*} p \delta_{3} \mathrm{e}^{-j z} \\
& \hat{u}_{z 0}=A_{1}^{*} c \mathrm{e}^{-c z}+A_{2}^{*} d \mathrm{e}^{-d z}+A_{3}^{*} p \mathrm{e}^{-j z} \\
& \hat{u}_{r 1}=A_{1}^{*} \gamma_{1} \mathrm{e}^{-c z}+A_{2}^{*} \gamma_{2} \mathrm{e}^{-d z}+A_{3}^{*} j \mathrm{e}^{-j z}
\end{aligned}
$$

$$
\hat{u}_{r 1}=A_{1}^{*} \eta_{1} \mathrm{e}^{-c z}+A_{2}^{*} \eta_{2} \mathrm{e}^{-d z}+A_{3}^{*} \delta_{3} j \mathrm{e}^{-j z}
$$

in which, $A_{1}^{*}, A_{2}^{*}$ and $A_{3}^{*}$ are arbitrary functions with respect to $p$, which can be determined by the boundary conditions of special problem.

\subsection{Boundary Conditions and Special Solutions}

Fig. 2 shows a schematic diagram for three-dimensional Lamb problem, where the ground surface is assumed to be permeable. The harmonic force, namely $-P_{0} \mathrm{e}^{\mathrm{i} \omega t}$, is acting on the ground surface at origin point along vertical direction. Assuming the force is acting within a circle plane with radius of $r_{0}$.

The vertical stress due to the force can be written as:

$$
\sigma_{z}(r)= \begin{cases}-\frac{P_{0}}{\pi r_{0}^{2}} & \left(r<r_{0}\right) \\ 0 & \left(r \geq r_{0}\right)\end{cases}
$$

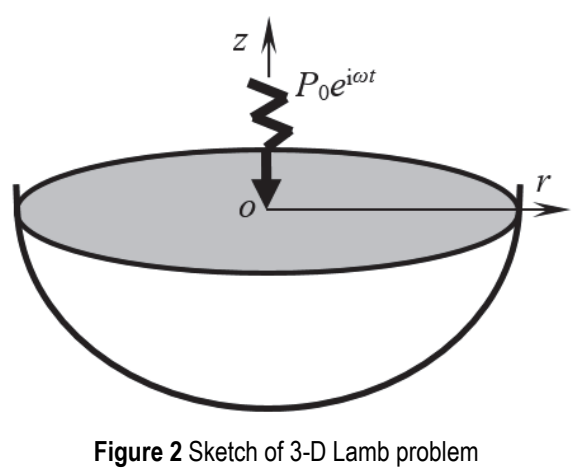

Applying zero-order Hankel transforms with respect to $r$ to Eq. (39) leads to:

$\hat{\sigma}_{z 0}=\int_{0}^{r 0} \sigma_{z}(r) r J_{0}(p r) \mathrm{d} r+\int_{r_{0}}^{+\infty} \sigma_{z}(r) r J_{0}(p r) \mathrm{d} r$

Obviously, when $r_{0} \rightarrow 0$, the force will translate into concentrated force. So:

$$
\hat{\sigma}_{z 0}=\lim _{r \rightarrow \infty} \int_{0}^{r} \frac{-P_{0}}{\pi r_{0}^{2}} r J_{0}(p r) \mathrm{d} r=-\frac{P_{0}}{2 \pi}
$$

This is Hankel transforms form for concentrated force $-P_{0} \mathrm{e}^{\mathrm{i} \omega t}$.

The boundary condition at depth $z=0$ can be given as follows:

$\hat{\tau}_{r z 1}=0$

$\hat{\sigma}_{z 0}=\frac{-P_{0}}{2 \pi}$

$\hat{p}_{f 0}=0$ 
Combining Eq. (29), Eq. (32) and Eq. (33), the arbitrary functions, namely $A_{1}^{*}, A_{2}^{*}$ and $A_{3}^{*}$ can be determined. Then the special solution for the Lamb problem can be given as:

$$
\begin{aligned}
& \hat{u}_{z 0}=\frac{P_{0}}{2 \pi D}\left(c a_{2}\left(p \gamma_{1}-j^{2}\right)+d a_{1}\left(j^{2}-p \gamma_{2}\right)\right) \\
& \hat{u}_{r 1}=\frac{P_{0}}{2 \pi D}\left(a _ { 1 } \left(\gamma_{2}\left(j^{2}+p^{2}\right)-d j\left(p+\gamma_{2}\right)+\right.\right. \\
& \left.+a_{2}\left(c j\left(p+\gamma_{1}\right)-\gamma_{1}\left(j^{2}+p^{2}\right)\right)\right)
\end{aligned}
$$

Finally, applying inverse Hankel transforms to Eq. (34), the displacement solutions in frequency domain can be obtained as:

$$
\begin{aligned}
& \bar{u}_{z}(r, 0)=\int_{0}^{+\infty} p \hat{u}_{z 0} J_{0}(p r) \mathrm{d} p \\
& \bar{u}_{r}(r, 0)=\int_{0}^{+\infty} p \hat{u}_{r 1} J_{1}(p r) \mathrm{d} p
\end{aligned}
$$

It should be noted that the three-dimensional solid skeleton displacement solutions, namely Eq. (35b), are originally derived in current work.

\section{VERIFICATION}

To verify the accuracy and effectiveness of the proposed solutions, verifications for both $2 \mathrm{D}$ and $3 \mathrm{D}$ scenarios are performed as follows:

\subsection{Verification for Two-Dimensional Solutions}

If there is no fluid in saturated soil, the saturated medium can be degenerated into a completely elastic single phase medium. Then, $\rho_{f}=0, n=0$ and $\alpha=0$, and the $\tilde{u}_{z}$ can be expressed as follows:

$$
\begin{aligned}
& \tilde{u}_{z}=-P_{0} \rho \omega^{2} \sqrt{p^{2}-\frac{\rho \omega^{2}}{\lambda+2 G}} /\left(4 G^{2} p^{4}-4 G p^{2} \omega^{2}-\right. \\
& \left.-\rho^{2} \omega^{4}-4 G^{2} p^{2} \sqrt{p^{2}-\frac{\rho \omega^{2}}{G}} \sqrt{p^{2}-\frac{\rho \omega^{2}}{\lambda+2 G}}\right)
\end{aligned}
$$

It should be noticed that $\frac{\lambda+2 G}{\rho}=v_{p}^{2}, \frac{G}{\rho}=v_{s}^{2}$, where $v_{p}, v_{s}$ denote the velocity of compressible wave and shear wave, respectively. Taking $k_{p}=\frac{\omega}{v_{p}}, k_{s}=\frac{\omega}{v_{s}}$, then Eq. (36) can be simplified as:

$$
\tilde{u}_{z}=-\frac{P_{0}}{G} k_{s}^{2} \sqrt{p^{2}-k_{p}^{2}} /\left(\left(2 p^{2}-k_{s}^{2}\right)^{2}-4 p^{2} \sqrt{p^{2}-k_{s}^{2}} \sqrt{p^{2}-k_{p}^{2}}\right)
$$

Applying inverse Fourier transforms to Eq. (37) leads

$$
\bar{u}_{z}=-\frac{P_{0}}{2 \pi G} \int_{-\infty}^{+\infty} \frac{k_{s}^{2} \sqrt{p^{2}-k_{p}^{2}} \mathrm{e}^{-\mathrm{i} p x}}{\left(\left(2 p^{2}-k_{s}^{2}\right)^{2}-4 p^{2} \sqrt{p^{2}-k_{s}^{2}} \sqrt{p^{2}-k_{p}^{2}}\right)} \mathrm{d} p
$$

The Eq. (38) is totally consistent with classical solution of Lamb problem in reference (Ewing et al., 1957) [25].

\subsection{Verification for Three-Dimensional Solutions}

Similarly, in three-dimensional elastic space the vertical displacement $\hat{u}_{z 0}$ solution can be derived by degeneration of Eq. (34a):

$$
\hat{u}_{z 0}=-\frac{P_{0}}{2 \pi G} \cdot \frac{k_{s}^{2} \sqrt{p^{2}-k_{p}^{2}}}{\left(2 p^{2}-k_{s}^{2}\right)^{2}-4 p^{2} \sqrt{p^{2}-k_{s}^{2}} \sqrt{p^{2}-k_{p}^{2}}}
$$

Applying inverse Hankel transforms to Eq. (39) gives:

$$
\bar{u}_{z}(r, 0)=-\frac{P_{0}}{2 \pi G} \cdot \int_{0}^{+\infty} \frac{p k_{s}^{2} \sqrt{p^{2}-k_{p}^{2}} J_{0}(p r)}{\left(2 p^{2}-k_{s}^{2}\right)^{2}-4 p^{2} \sqrt{p^{2}-k_{s}^{2}} \sqrt{p^{2}-k_{p}^{2}}} \mathrm{~d} p
$$

It can be observed that Eq. (40) is well consistent with the solution as presented in reference (Ewing et al, 1957).

In order to further verify the accuracy of the presented 3D solutions, comparison studies are also conducted between the results obtained from the proposed solutions (Eq. (35a)) and the approach by Sharma (1992)[18] for Lamb problem in saturated porous media. The physical and mechanical parameters used in the case are given in Tab.1. A harmonic load in $z$ direction with load frequency $\omega$ of 30 $\mathrm{rad} / \mathrm{s}$ and amplitude $P_{0}$ of $10^{3} \mathrm{~Pa}$ is acting on the half-space ground. The results of vertical displacement, including real part and imaginary part, in terms of radical distance are given in Fig. 3. A very good agreement is observed between the results based on Sharma's formula and those from Eq. (35a), which further highlights the effectiveness and accuracy of the proposed solutions in present work.
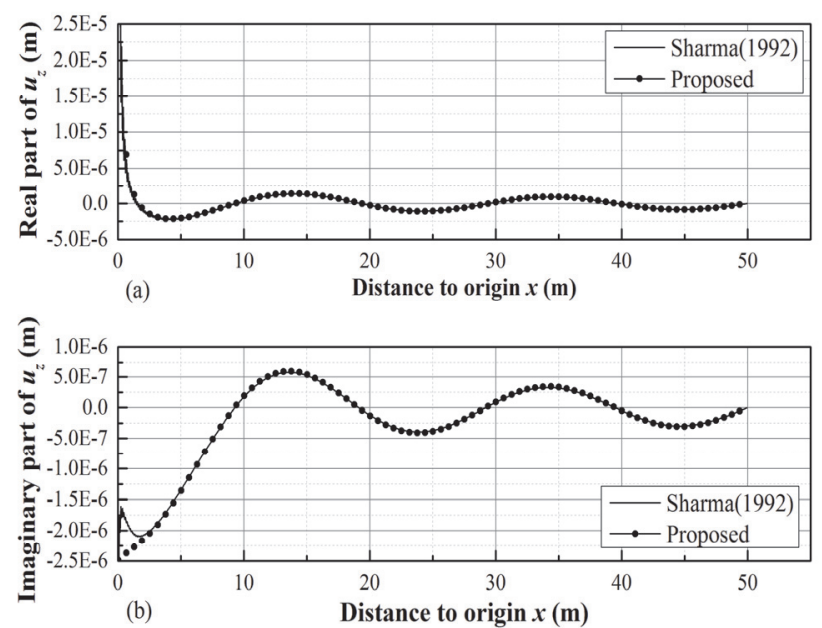

Figure 3 Comparing solid skeleton vertical displacement solutions from Sharma with those proposed in the paper (a) Real part of uz-x curves, (b) Imaginary part of uz-x curves to: 


\section{PARAMETRIC STUDY FOR SOLID SKELETON DISPLA- CEMENT IN 3D PROBLEM}

\subsection{Non-Dimensional Governing Equations and Displace-} ment Solutions

Prior to parametric study, the solid skeleton displacement solutions for three-dimension against distance to origin are firstly discussed in detail herewith. For the sake of discussion without losing its generality, the non-dimensional governing equations are given firstly.

Taking the non-dimensional parameters as shown below:

$$
\begin{array}{rlrl}
G^{*} & =\frac{G}{\lambda_{c}+2 G} ; \lambda_{c}^{*}=\frac{\lambda_{c}}{\lambda_{c}+2 G} ; M^{*} & =\frac{M}{\lambda_{c}+2 G} \\
\rho^{*} & =1.0 ; \quad \kappa^{*}=1.0 ; \quad \rho_{f}^{*}=\frac{\rho_{f}}{\rho} ;
\end{array}
$$

and non-dimensional coordinates and time presented as follows:

$$
\xi=\frac{x}{\rho \kappa V_{c}} ; \eta=\frac{y}{\rho \kappa V_{c}} ; \zeta=\frac{z}{\rho \kappa V_{c}} ; \tau=\frac{t}{\rho \kappa}
$$

where $V_{c}=\sqrt{\left(\lambda_{c}+2 G\right) / \rho}$, Eq. (1) can be written as:

$$
\begin{aligned}
& G^{*} \boldsymbol{u}_{i, j j}+\left(\lambda_{c}^{*}+G^{*}\right) \boldsymbol{u}_{j, j i}+\alpha M^{*} \boldsymbol{w}_{j, j i}=\rho^{*} \ddot{\boldsymbol{u}}_{i}+\rho_{f}^{*} \ddot{\boldsymbol{w}}_{i} \\
& \alpha M^{*} \boldsymbol{u}_{j, j i}+M^{*} \boldsymbol{w}_{j, j i}=\rho^{*} \ddot{\boldsymbol{u}}_{i}+\frac{\rho_{f}^{*}}{n} \ddot{\boldsymbol{w}}_{i}+\frac{1}{\kappa} \dot{\boldsymbol{w}}_{i}
\end{aligned}
$$

where the single point and double point above the letter denote the first-order and second-order derivation with regard to $\tau$; the subscript $i, j$ equal to 1,2 , or 3 and represent $\xi, \eta, \zeta$ respectively.

It is should be noted that Eq. (1) and Eq. (43) have the same structure. Therefore, for three-dimensional Lamb problem, the general solutions for the Eq. (43) have the same structure of Eq. (29) once parameters in Eq. (29) are replaced by corresponding non-dimensional parameters given in Eq. (41). The boundary conditions basically keep unchanged except Eq. (33b) should be written as:

$$
\hat{\sigma}_{z 0}^{*}=\frac{-P_{0}}{2 \pi} \cdot \frac{1}{\rho V_{c}^{2}}
$$

Analogously, the special solutions for the Lamb problem with non-dimensional parameters should have the same structure as Eq. (34). Therefore, they can be written as:

$$
\hat{u}_{z 0}=\frac{P_{0}}{2 \pi D^{*} \rho V_{c}^{2}}\left(c^{*} a_{2}^{*}\left(p \gamma_{1}^{*}-\left(\left(j^{* 2}\right)\right)+d^{*} a_{1}^{*}\left(\left(j^{*}\right)^{2}-p \gamma_{2}^{*}\right)\right)\right.
$$

$$
\begin{aligned}
& \hat{u}_{r 1}=\frac{P_{0}}{2 \pi D \rho V_{c}^{2}}\left(a_{1}^{*}\left(\gamma_{2}^{*}\left(\left(j^{*}\right)^{2}+p^{2}\right)-d^{*} j^{*}\left(p+\gamma_{2}^{*}\right)\right)+\right. \\
& +a_{2}^{*}\left(c^{*} j^{*}\left(p+\gamma_{1}^{*}\right)-\gamma_{1}^{*}\left(\left(\left(j^{*}\right)^{2}+p^{2}\right)\right)\right)
\end{aligned}
$$

where the parameters with asterisk have the same structure as those without asterisk. Finally, for 3D Lamb problem, the displacement solutions $\bar{u}_{z}(r, 0)$ and $\bar{u}_{r}(r, 0)$ can be given by inverse Hankel transforms based on Eq. (45). It is noted that, in present work, non-dimensional displacement solutions are derived as follows:

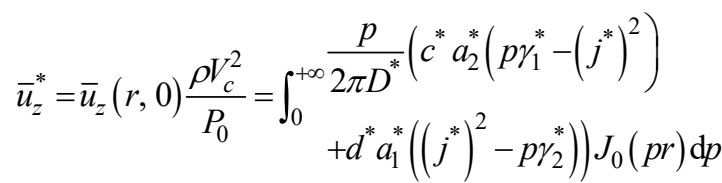

$$
\begin{aligned}
& \bar{u}_{r}^{*}= \bar{u}_{r}(r, 0) \frac{\rho V_{c}^{2}}{P_{0}}= \\
&=\int_{0}^{+\infty} \frac{p}{2 \pi D^{*}}\left(a _ { 1 } ^ { * } \left(\gamma_{2}^{*}\left(\left(j^{*}\right)^{2}+p^{2}\right)\right.\right. \\
&\left.\quad-d^{*} j^{*}\left(p+\gamma_{2}^{*}\right)\right)+a_{2}^{*}\left(c^{*} j^{*}\left(p+\gamma_{1}^{*}\right)\right. \\
&\left.\left.-\gamma_{1}^{*}\left(\left(j^{*}\right)^{2}+p^{2}\right)\right)\right) J_{1}(p r) \mathrm{d} p
\end{aligned}
$$

\subsection{Parametric Study}

In order to investigate the effects of the governing parameters (i.e., exciting frequency, bulk modulus of soil matrix, and bulk modulus of pore fluid) on variation of nondimensional displacement with the increasing distance away from the excitation source, a series of parametric studies are conducted.
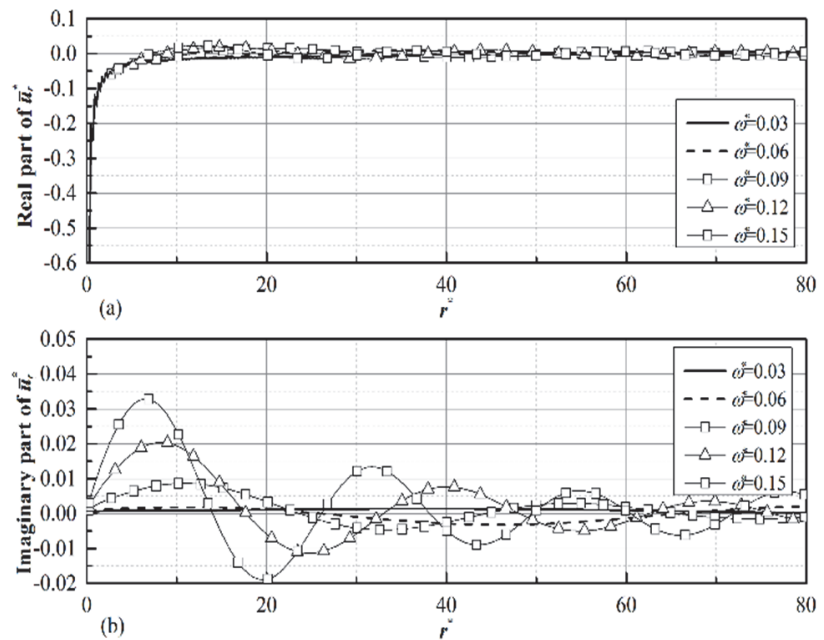

Figure 4 The influence of non-dimensional load exciting frequency $\omega^{*}$ on solid skeleton horizontal displacements in 3-D Lamb problem (a) Real part of horizontal displacements $\bar{u}_{r}^{*}-r^{*}$ curves (b) Imaginary part of horizontal displac

$$
\text { ments } \bar{u}_{r}^{*}-r^{*} \text { curves }
$$



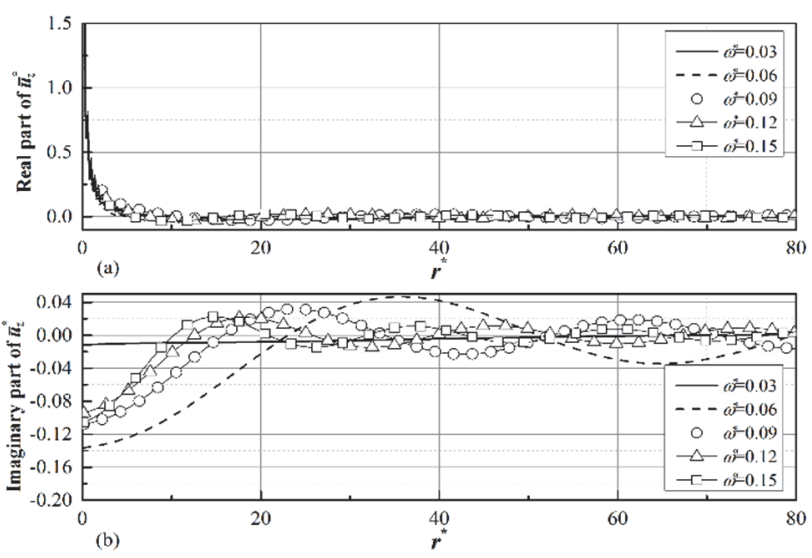

Figure 5 The influence of non-dimensional load exciting frequency $\omega^{*}$ on vertical displacements of solid skeleton for 3-D Lamb problem (a) Real part of horizontal displacements $\bar{u}_{z}^{*}-r^{*}$ curves (b) Imaginary part of horizontal displace-

$$
\text { ments } \bar{u}_{z}^{*}-r^{*} \text { curves }
$$

In the studied case, a harmonic concentrated load is acting on the ground surface with permeable boundary condition. The physical and mechanical parameters used are given in Tab. 1. Meanwhile, non-dimensional parameters are given in Tab. 2, where $K_{f}^{*}$ are defined as $K_{f} /\left(\lambda_{c}+2 G\right)$. The non-dimensional exciting frequency can be inferred as $\omega^{*}=\rho \kappa \omega$. According to Biot's theory, the governing equations Eq. (1) are applicable for the excitation at low frequency domain. That is, the frequency is lower than the cut-off frequency as follows:

$$
\omega \leq 0.154 \frac{n}{\rho_{f} \kappa}
$$

Table 1 Physical and mechanical parameters for the saturated soil.

\begin{tabular}{|l|l|l|l|l|l|l|l|l|}
\hline$G / \mathrm{Pa}$ & $v$ & $\alpha$ & $M / \mathrm{Pa}$ & $\begin{array}{l}k_{d} / \\
\mathrm{m} / \mathrm{s}\end{array}$ & $n$ & $\begin{array}{l}\rho / \\
\mathrm{kg} / \mathrm{m}^{3}\end{array}$ & $\begin{array}{l}\rho_{f} / \\
\mathrm{kg} / \mathrm{m}^{3}\end{array}$ & $K_{f} / \mathrm{Pa}$ \\
\hline $\begin{array}{l}1.94 \times \\
10^{7}\end{array}$ & 0.2 & 0.6 & $\begin{array}{l}6.31 \times \\
10^{6}\end{array}$ & $10^{-2}$ & 0.6 & 1680 & 1000 & $3.79 \times 10^{6}$ \\
\hline
\end{tabular}

Table 2 Non-dimensional parameters considered
\begin{tabular}{|l|l|l|l|l|l|l|l|l|}
\hline$G^{*}$ & $v$ & $\alpha$ & $M^{*}$ & $\kappa^{*}$ & $n$ & $\rho^{*}$ & $\rho_{f}^{*}$ & $K_{f}^{*}$ \\
\hline 0.36 & 0.2 & 0.60 & 0.12 & 1.0 & 0.6 & 1.0 & 0.60 & 0.07 \\
\hline
\end{tabular}
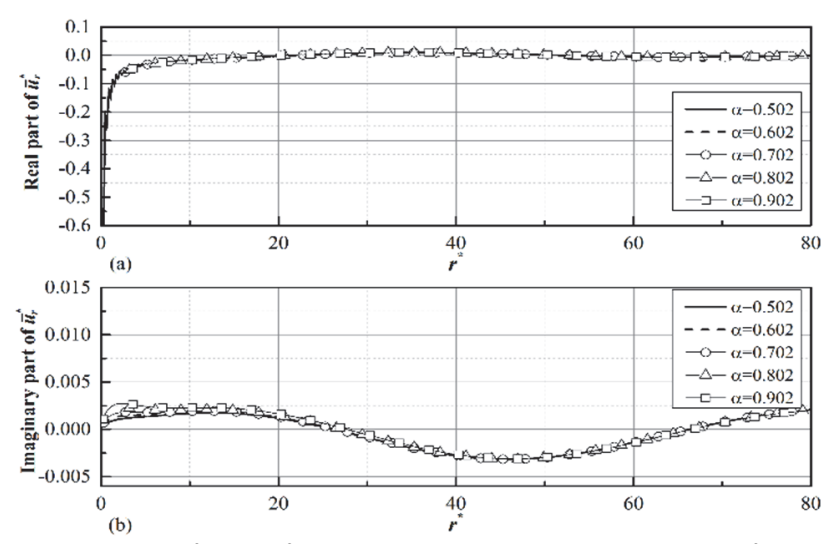

Figure 6 The influence of parameter $\alpha$ on horizontal displacements of solid skeleton in 3D Lamb problem (a) Real part of horizontal displacements $\bar{u}_{r}^{*}-r^{*}$ curves (b) Imaginary part of horizontal displacements $\bar{u}_{r}^{*}-r^{*}$ curves
Thus, the upper bound of excitation frequency is determined as $92.4 \mathrm{rad} / \mathrm{s}$, with the corresponding non-dimensional value $\omega^{*}$ of 0.155 .

Fig. 4 and Fig. 5 present the variation of displacements of solid skeleton along the horizontal and vertical direction, respectively, with the increasing distance from the excitation source with the non-dimensional frequency $\omega^{*}$ varying from 0.03 to 0.15 . It is noted that the horizontal axis represents non-dimensional radical distance $r^{*}=r /\left(\rho \kappa V_{c}\right)$. It is observed that, for real part of ground displacements of solid skeleton in both horizontal and vertical directions, the effect of non-dimensional excitation frequency $\omega^{*}$ on the variation of non-dimensional displacement against the distance from excitation source is insignificant. In contrast, for imaginary part of horizontal displacements, an increase in $\omega^{*}$ leads to a significant increase in the amplitude of horizontal displacement of solid skeleton, but this effect would decrease gradually with the increasing distance away from the excitation source. It is found that the maximum imaginary amplitude occurs when frequency $\omega^{*}$ is taken the value 0.06 . Also, the effect of $\omega^{*}$ on the amplitude of vertical displacement of solid skeleton is relatively insignificant.
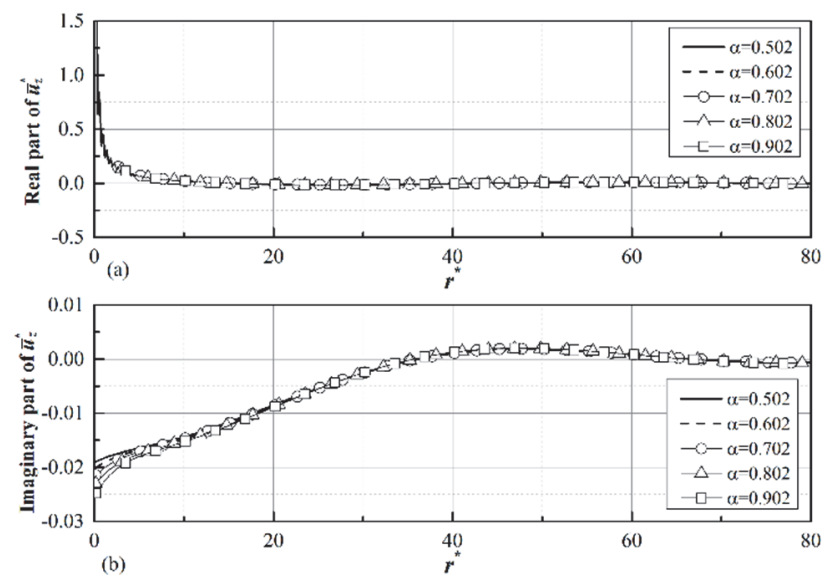

Figure 7 The influence of parameter $\alpha$ on vertical displacements of solid skeleton in 3D Lamb problem (a) Real part of horizontal displacements $\bar{u}_{z}^{*}-r^{*}$ curves (b) Imaginary part of horizontal displacements $\bar{u}_{z}^{*}-r^{*}$ curves

Fig. 6 and Fig. 7 present the displacements of solid skeleton along horizontal and vertical direction, respectively, with the increasing distance from the excitation source corresponding to the parameter $\alpha$ varying from 0.502 to 0.902 . Note that, based on the definition of parameter $\alpha$, the greater the parameter $\alpha$ is, the larger the bulk modulus of soil matrix is if the bulk modulus of soil skeleton keeps unchanged.

In this study, the other parameters (except $\alpha$ ) in Tab. 2 remain constant. As observed in Fig. 6 and Fig. 7, it can be found that the variation of the displacements of solid skeleton along both horizontal and vertical direction is insensitive to the change in the value of $\alpha$ (i.e., bulk modulus of soil matrix).

Fig. 8 and Fig. 9 present the displacements of solid skeleton along horizontal and vertical direction, respecti- 
vely, with the increasing distance from the excitation source corresponding to the bulk modulus $K_{f}^{*}$ of pore fluid varying from 0.07 to 1.93 . Similarly, in the analysis, the other parameters (except $K_{f}^{*}$ ) in Tab. 2 remain constant. It can be observed that, for bulk modulus of pore fluid $K_{f}^{*}<$ 1.3 , the effect of $K_{f}^{*}$ on the ground displacements in both horizontal and vertical directions is insignificant. Nevertheless, when the bulk modulus $K_{f}^{*}$ is greater than 1.3 , the increase of $K_{f}^{*}$ would lead to significant increase in the ground displacements in both horizontal and vertical direction, which highlights that the compressibility of the pore fluid should be carefully considered for evaluating the ground surface displacements.
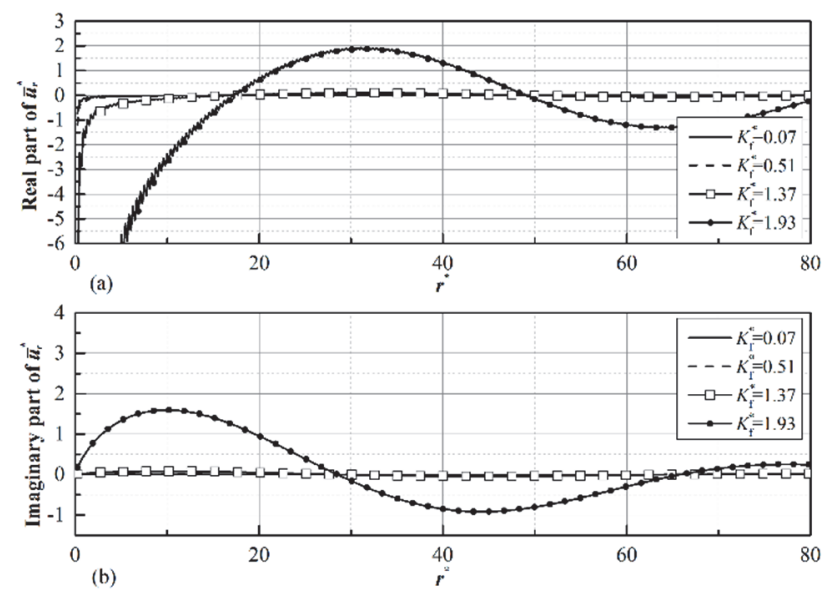

Figure 8 The influence of non-dimensional parameter $K_{f}^{*}$ on horizontal displacements of solid skeleton in 3D Lamb problem (a) Real part of horizontal displacements $\bar{u}_{r}^{*}-r^{*}$ curves (b) Imaginary part of horizontal displacements

$$
\bar{u}_{r}^{*}-r^{*} \text { curves }
$$
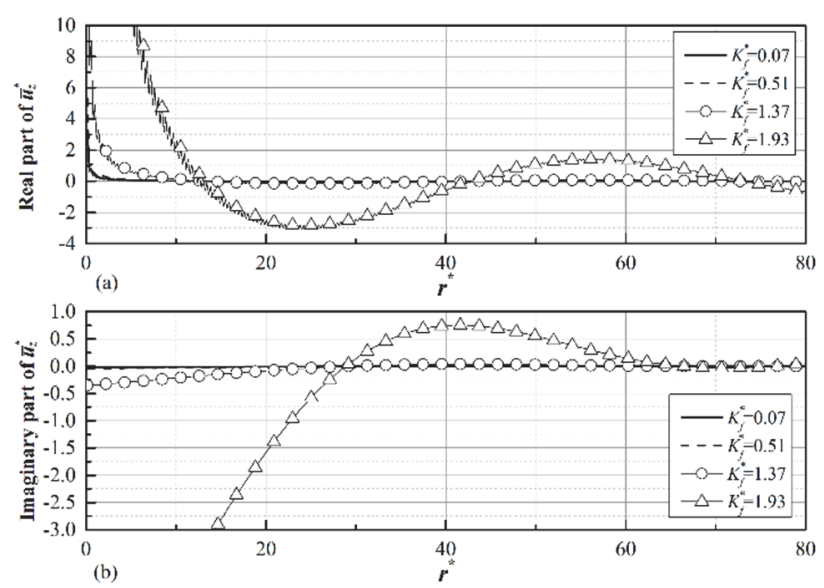

Figure 9 The influence of non-dimensional parameter $K_{f}^{*}$ on vertical displacements of solid skeleton in 3D Lamb problem (a) Real part of horizontal displacements $\bar{u}_{z}^{*}-r^{*}$ curves (b) Imaginary part of horizontal displacements $\bar{u}_{z}^{*}-r^{*}$ curves

\section{CONCLUSIONS}

In this paper, to avoid the complexity, a simple and efficient analytical solution is derived for both $2 \mathrm{D}$ and $3 \mathrm{D}$
Lamb problems in saturated soil under harmonic excitation. In addition, unlike the existing solutions, the proposed solutions for both 2D and 3D Lamb problems in saturated soil under harmonic excitation are capable of well revealing the effect of compressibility of both liquid phase and solid phase on the ground displacements firstly. Verifications are conducted by comparing the degeneration results obtained from the proposed solutions with those obtained from existing solutions. For the sake of discussion without losing its generality, the non-dimensional solutions for three-dimensional Lamb problem are also derived. Furthermore, parametric studies on displacements on ground surface in both horizontal and vertical direction are carried out.

The results show that the exciting frequency and compressibility of the pore fluid are the governing factors for the variation of ground displacements, while the effect of compressibility of soil matrix on the ground displacements is insignificant, which highlights that the compressibility of the pore fluid should be carefully considered for evaluating the movements of ground when it is subjected to excitations.

The integral transformation, which is simple and reliable is utilized for solving in the paper. In addition, in the process of parameter study, the dimensionless strategy is adopted to avoid the influence of physical dimension on the calculation results, which makes the calculation results more general. These methods are all effective theoretical analysis methods. However, it should be pointed out that the methods in the paper should deal with numerical inverse transformation, which often requires some numerical calculation skills.

\section{Acknowledgment}

This work is supported by the National Natural Science Foundation of China (No. 51478289).

\section{REFERENCES}

[1] Lamb, H. (1904). On the propagation of tremors over the surface of an elastic solid. Philosophical Transactions of The Royal Society, 203(359-371), 1-42. https://doi.org/10.1098/rsta.1904.0013

[2] Liu, G. (2007). Exact solution for a two-dimensional Lamb's problem due to a strip impulse. Acta Mechanica Solida Sinica, 20(3), 258-265. https://doi.org/10.1007/s10338-007-0730-0

[3] Han, J. H. \& Kim, Y. J. (2015). Time-frequency beamforming for nondestructive evaluations of plate using ultrasonic Lamb wave. Mechanical Systems and Signal Processing, 2015(54-55), 336-356. https://doi.org/10.1016/j.ymssp.2014.09.008

[4] Cagniard, L., Flinn, E. A., \& Dix, C. H. (1962). Reflection and Refraction of progressive Seismic Waves. McGraw-Hill, New York, U.S.A

[5] de Hoop, A. T. (1960). Modification of Cagniard's method for solving seismic pulse problems. Applied Scientific Research, 8(1), 349-356. https://doi.org/10.1007/BF02920068

[6] Liu, G. (2007). Exact solution for a two-dimensional Lamb's problem due to a strip impulse. Acta Mechanica Solida Sinica, 20(3), 258-265. https://doi.org/10.1007/s10338-007-0730-0 
[7] Kravtsov, A. V., Kuznetsov, S. V., Sekerzh-Zen'kovich, S. Y. (2011). Finite element models in Lamb's problem. Mechanics of Solids, 46(6), 952-959. https://doi.org/10.3103/S002565441106015X

[8] Guenfoud, S., Amrane, M. N., Bosakov, S. V., et al. (2009). Semi-analytical evaluation of integral forms associated with Lamb's problem. Soil Dynamics \& Earthquake Engineering, 29(3), 438-443. https://doi.org/10.1016/j.soildyn.2008.04.007

[9] Arcos, R., Romeu, J., Clot, A., et al. (2013). Some analytical aspects of viscoelastic Lamb's problem for improving its numerical evaluation. Wave Motion, 50(2), 226-232. https://doi.org/10.1016/j.wavemoti.2012.08.010

[10] Kraut, E. A. (1963). Advances in the theory of anisotropic elastic wave propagation. Reviews of Geophysics, 1(3), 401448. https://doi.org/10.1029/RG001i003p00401

[11] Burridge, R. (1970). The directions in which Rayleigh waves may be propagated on crystals. Quarterly Journal of Mechanics \& Applied Mathematics, 23(2), 217-224. https://doi.org/10.1093/qjmam/23.2.217

[12] Willis, J. R. (1973). Self-Similar problems in elastodynamics. Philosophical Transactions of the Royal Society of London, 274(1240), 435-491. https://doi.org/10.1098/rsta.1973.0073

[13] Willis, J. R. \& Bedding, R. J. (1975). Arrivals associated with a class of self-similar problems in elastodynamics. Mathematical Proceedings of the Cambridge Philosophical Society, 77, 591-607. https://doi.org/10.1017/S0305004100051410

[14] Wang, C. Y., Achenbach, J. D. (1996). Lamb's problem for solids of general anisotropy. Wave Motion, 24(3), 227-242. https://doi.org/10.1016/S0165-2125(96)00016-9

[15] Paul, S. (1976). On the displacements produced in a porous elastic half-space by an impulsive line load (Non-Dissipative Case). Pure \& Applied Geophysics, 114(4), 605-614. https://doi.org/10.1007/BF00875654

[16] Paul, S. (1976). On the disturbance produced in a semi-infinite poroelastic medium by a surface load. Pure \& Applied Geophysics, 114(4), 615-627. https://doi.org/10.1007/BF00875655

[17] Philippacopoulos, A. J. (1988). Lamb's problem for fluid-saturated porous media. Bulletin of the Seismological Society of America, 78(2), 908-923

[18] Sharma, M. D. (1992). Comments on Lamb's problem for fluid-saturated porous media. Bulletin of the Seismological Society of America, 82(5), 2263-2273.

[19] Huang, Y., Wang, X. G. (2004). The 3-D Non-axisymmetrical Lamb problem in transversely isotropic saturated poroelastic media. Science in China, 34(9), 1037-1060. https://doi.org/10.1360/03ye0331

[20] Wang, X. G. (2011). Three-dimensional transient Lamb's problem of transversely isotropic saturated soils. Rock and soil Mechanics, 32(1), 253-260.

[21] Mattos, H. S. D. C., Teixeira, L. P., \& Martins-Costa, M. L. (2018). Analysis of small temperature oscillation in a deformable solid matrix containing a spherical cavity filled with a compressible liquid-Analytical solutions for damage initiation induced by pore pressure variation. International Journal of Engineering Science, 129, 1-20. https://doi.org/10.1016/j.ijengsci.2018.02.014

[22] Ai, Z. Y. \& Hu, Y. D. (2016). The analysis of beams on layered poroelastic soils with anisotropic permeability and compressible pore fluid. Applied Mathematical Modelling, 40(11-12), 5876-5890. https://doi.org/10.1016/j.apm.2016.01.032

[23] Tamellini, M., Parolini, N., \& Verani, M. (2018). An optimal control problem for two-phase compressible incompressible flows. Computers and Fluids, 172, 538-548. https://doi.org/10.1016/j.compfluid.2018.03.039
[24] Biot, M. A. (1956). Theory of propagation of elastic waves in a fluid-saturated porous solid : low frequency rang. Journal of the Acoustical Society of America, 44(2), 168-178. https://doi.org/10.1121/1.399081

[25] Ewing, W. M, Jardetzky W. S. (1957). Elastic Waves in Layered Media. McGraw-Hill, New York, U.S.A. https://doi.org/10.1063/1.3060203

\section{Contact information:}

\section{Hao XIONG}

(Corresponding author)

School of Civil Engineering and Architecture,

Taizhou University,

No. 1139, Shifu Avenue,

Taizhou City, Zhejiang Province, 318000, China

E-mail: haosyong@126.com

\section{Qingsheng CHEN}

School of Civil, Architecture and Environmental Engineering, Hubei University of Technology, Wuhan 430068, China

\section{Xiaogang WANG}

School of Civil Engineering and Architecture, Taizhou University, Taizhou, 318000, China 